\title{
Using magnetic activity and Galactic dynamics to constrain the ages of $M$ dwarfs
}

\author{
Andrew A. West ${ }^{1}$, Suzanne L. Hawley ${ }^{2}$, John J. Bochanski ${ }^{1}$, \\ Kevin R. Covey ${ }^{3}$ and Adam J. Burgasser ${ }^{1}$ \\ ${ }^{1}$ MIT Kavli Institute for Astrophysics and Space Research, \\ 77 Massachusetts Ave, Cambridge, MA 02139-4307 \\ email: aaw@mit.edu, jjb@mit.edu, ajb@mit.edu \\ ${ }^{2}$ Department of Astronomy, University of Washington, \\ Box 351580, Seattle, WA 98195 \\ email: slh@astro.washington.edu \\ ${ }^{3}$ Harvard-Smithsonian Center for Astrophysics, \\ 60 Garden Street, Cambridge MA 02138 \\ email: kcovey@cfa.harvard.edu
}

\begin{abstract}
We present a study of the dynamics and magnetic activity of $\mathrm{M}$ dwarfs using the largest spectroscopic sample of low-mass stars ever assembled. The age at which strong surface magnetic activity (as traced by $\mathrm{H} \alpha$ ) ceases in $\mathrm{M}$ dwarfs has been inferred to have a strong dependence on mass (spectral type, surface temperature) and explains previous results showing a large increase in the fraction of active stars at later spectral types. Using spectral observations of more than 40000 M dwarfs from the Sloan Digital Sky Survey, we show that the fraction of active stars decreases as a function of vertical distance from the Galactic plane (a statistical proxy for age), and that the magnitude of this decrease changes significantly for different $M$ spectral types. Adopting a simple dynamical model for thin disk vertical heating, we assign an age for the activity decline at each spectral type, and thus determine the activity lifetimes for $\mathrm{M}$ dwarfs. In addition, we derive a statistical age-activity relation for each spectral type using the dynamical model, the vertical distance from the Plane and the $\mathrm{H} \alpha$ emission line luminosity of each star (the latter of which also decreases with vertical height above the Galactic plane).
\end{abstract}

Keywords. stars: activity, stars: kinematics, stars: late-type, stars: low-mass, brown dwarfs

\section{Introduction}

M dwarfs are the smallest and least luminous stars on the main sequence, yet they are the most numerous of all stellar constituents and have lifetimes longer than the current age of the Universe. Determining the age of an individual main sequence, field M dwarf is quite challenging. Stellar clusters would seem to provide the ideal environments for calibrating the ages of $M$ dwarfs (because the age of the cluster is determined). However, the large distances to clusters older than a few Gyr (and resulting faintness of cluster M dwarfs), preclude any detailed observations of the intermediate or oldest cluster resident $\mathrm{M}$ dwarfs. Age estimates for field $\mathrm{M}$ dwarfs must therefore rely on a number of techniques that piggyback on other age dating methods. Although several methods exist for estimating the ages of $\mathrm{M}$ dwarfs (e.g. contribution by S. Catalan), we focus this contribution on what the magnetic activity of $\mathrm{M}$ dwarfs can tell us about their age.

$\mathrm{M}$ dwarfs are host to intense magnetics dynamos that give rise to chromospheric and coronal heating, producing emission from the X-ray to the radio. This magnetic activity has long been thought to be tied to the age of the host star. Almost 40 years ago, Wilson 
\& Woolley (1970) found a link between magnetic activity (as traced by the Ca II H \& K emission lines) and the orbital elements (namely the inclination and eccentricity) of more than 300 late-type dwarfs; stars in near-circular orbits with small inclinations had the strongest activity. They concluded that as stars age, their orbits get more inclined and more eccentric (due to dynamical interactions), and thus, magnetic activity must also decline with age. Subsequent studies over the following decades have found similar connections between age and activity in low-mass stars (Wielen 1977; Giampapa \& Liebert 1986; Soderblom et al. 1991; Hawley et al. 1996, Hawley et al. 1999, Hawley et al. 2000).

Although activity has been observed for decades, the exact mechanism that gives rise to the chromospheric heating is still not well-understood. In the Sun, magnetic field generation and subsequent heating is closely tied to the Sun's rotation. From helioseismology, we know that there is a rotational boundary between the inner solid-body rotating radiative zone and the outer differentially rotating convective zone (Parker 1993; Ossendrijver 2003; Thompson et al. 2003). This boundary, dubbed the tachocline, creates a rotational sheer that likely allows magnetic fields to be generated, preserved and eventually rise to the surface where they emerge as magnetic loops. These loops bring heat to the Sun's chromosphere and corona, driving both large stellar flares, as well as lower-level quiescent (or persistent) magnetic activity. The faster a star (with a tachocline) rotates, the stronger its magnetic heating and surface activity. Therefore, the angular momentum evolution of solar-type stars should play an important role in determining the magnitude of the observed activity.

Angular momentum loss from magnetized winds has been shown to slow rotation in solar-type stars; as a result magnetic activity decreases. Skumanich (1972) found that both activity and rotation decrease over time as a power law $\left(t^{-0.5}\right)$. Subsequent studies confirmed the Skumanich results and empirically demonstrated a strong link between age, rotation and activity in solar-type stars (Barry 1988; Soderblom et al. 1991; Pizzolato et al. 2003; Mamajek \& Hillenbrand 2008; see contribution by E. Mamajek).

There is strong evidence that the rotation-activity (and presumably age) relation extends from stars more massive than the Sun to smaller dwarfs (Pizzolato et al. 2003; Mohanty \& Basri 2003; Kiraga \& Stepien 2007). Therefore, rotation derived ages (using gyrochronology) may provide a useful independent estimate of age for $M$ dwarfs when large enough calibration samples can be acquired (see contributions by S. Meibom, J. Irwin, and S. Barnes). However, at a spectral type of $\sim \mathrm{M} 3\left(0.35 \mathrm{M}_{\odot}\right.$; Reid \& Hawley 2005; Chabrier \& Baraffe 1997), stars become fully convective and the tachocline presumably disappears. Despite this changes, magnetic activity persists in late-type M dwarfs; the fraction of active $\mathrm{M}$ dwarfs peaks around a spectral type of $\mathrm{M} 7$ before decreasing into the brown dwarf regime (Hawley et al. 1996; Gizis et al. 2000; West et al. 2004).

It is unclear if the rotation-age-activity relation extends to the fully convective $M$ dwarfs. A few empirical studies have uncovered evidence that activity and rotation might be linked in late-type M dwarfs (Delfosse et al. 1998; Mohanty \& Basri 2003; Reiners \& Basri 2007). In addition, recent simulations of fully convective stars find that rotation may play a role in magnetic dynamo generation (Dobler et al. 2006; Browning 2008). However, a recent study found detectable rotation in a few inactive M7 dwarfs, indicating that the situation may in fact be more complicated (West \& Basri 2009).

Irrespective of rotation, many studies have found evidence that the age-activity relation extends into the M dwarf regime. Eggen (1990) observed a Skumanich-type decrease in activity as a function of age. Larger samples of $\mathrm{M}$ dwarfs have added further evidence that magnetic activity decreases with age (Fleming et al. 1995; Gizis et al. 2002). There is also evidence that M dwarfs may have finite active lifetimes. Stauffer et al. (1994) suggested that activity may not be present in the most massive $M$ dwarfs in the Pleiades. Hawley 
et al. (2000) confirmed the Stauffer et al. (1994) claim by observing a a sample of clusters that spanned several Gyr in age. They were able to calibrate the lifetimes for early-type $\mathrm{M}$ dwarfs by observing the color at which activity (traced by $\mathrm{H} \alpha$ emission) was no longer present. Because of the small sample size, the derived Hawley et al. (2000) activity ages are lower limits at a given color or spectral type. As mentioned above, the Hawley et al. (2000) study was limited to younger, nearby clusters due to the intrinsic faintness of M dwarfs; thus, it could only probe ages of a few Gyr and spectral types as late as $\sim \mathrm{M} 3$. Larger samples of $\mathrm{M}$ dwarfs are required to statistically derive age-activity relations that extend both in the ages they probe and the range of spectral types they cover.

In this contribution, we review recent studies that have used large spectroscopic samples to investigate the relationship between age and activity in $\mathrm{M}$ dwarfs. In addition, we derive a statistical $\mathrm{H} \alpha$ age-activity relation for M2-M7 dwarfs.

\section{SDSS DR5 low-mass star spectroscopic sample}

Large surveys such as the Sloan Digital Sky Survey (SDSS; York et al. 2000) have created optical and infrared catalogs of tens of millions of $\mathrm{M}$ dwarfs (Bochanski et al. 2009 in prep). In addition to the photometric data, the SDSS has obtained spectra for tens of thousands of M dwarfs. Recently, these surveys have been utilized to examine the statistical properties of M dwarfs, the dynamics of the Milky Way and detailed studies of magnetic activity (Hawley et al. 2002; West et al. 2004; West et al. 2006; Covey et al. 2007; Bochanski et al. 2007a, 2007b; West et al. 2008; hereafter W08).

Recently, the SDSS Data Release 5 (DR5) low-mass star spectroscopic sample was released (W08) $\dagger$, containing over $44000 \mathrm{M}$ and early L-type dwarfs. Radial velocities (accurate to within $\sim 5 \mathrm{~km} \mathrm{~s}^{-1}$ ) and photometric distances (from the $r-z$ colors; see Bochanski et al. 2009 in prep) were measured for all of the stars. Proper motions (matches to USNO-B; Munn et al. 2004) were obtained for over 27000 of the stars, allowing for full $U, V, W 3$-D space motions for over 27000 low-mass dwarfs, the largest such sample ever assembled. In addition, all of the spectra were run through the Hammer spectral typing facility (Covey et al. 2007) to assign spectral types and measure the strength of the $\mathrm{H} \alpha$ emission line. One of the hallmarks of the W08 sample is that it probes the entirety of the Galactic thin disk and extends well into the thick disk ( $\sim 50-2500$ pc above the Plane). This is due to the fact that the majority of SDSS fields are in the North Galactic Cap and that SDSS was designed to study the distant Universe; intrinsically faint Galactic $\mathrm{M}$ dwarfs are bright in a deep extragalactic survey.

\section{Results}

\subsection{H $\alpha$ activity}

Activity in $M$ dwarfs varies as a function of spectral type. Figure 1 shows the fraction of active M dwarfs as a function of spectral type from West et al. (2004). The active fraction is very small for early-type $\mathrm{M}$ dwarfs, peaks at M7-M8 and declines into the $\mathrm{L}$ dwarf regime. While some of the morphology of this relation may be due to the ability (or inability) to host a strong dynamo (the activity fraction decrease in the late-type $\mathrm{M}$ and L dwarfs is likely caused by the atmospheres becoming neutral), a large part of the shape may be due to age effects. If activity has a finite lifetime that changes as a function

$\dagger$ Measured quantities can be obtained electronically using the CDS Vizier database http://vizier.u.strasbg.fr/vis-bin/VizieR 
of spectral type, and we observe a range of stellar ages, then stars with longer lifetimes will appear to have higher activity fractions.

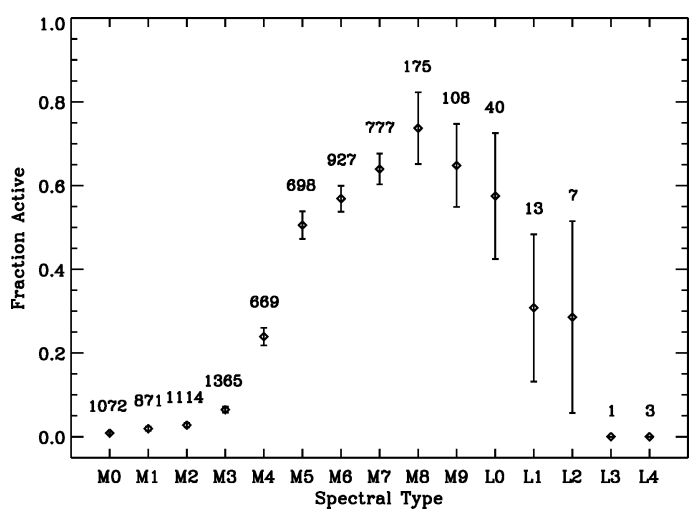

Figure 1. Fraction of active stars as a function of spectral type (reproduced from West et al. 2004). Numbers above each point represent the number of stars used to compute the fraction.

To test this hypothesis, West et al. $(2004,2006)$ showed that the fraction of active M7 stars decreases as a function of vertical distance from the Galactic Plane (see Figure 2). Stars are born in dynamically cold molecular gas near the midplane of the Galaxy. Over time they undergo dynamical interactions with molecular clouds, which add energy to the stellar orbits in all dimensions (see contribution by B. Nordström). It is this process that gives thickness to the Milky Way disk and allows us to use vertical distance as a proxy for age; stars further from the Plane are statistically older (they have to be dynamically heated to reach those heights), while stars near the Plane are statistically younger. Figure 2 shows that the younger stars, near the plane of the Milky Way are almost all active, whereas the fraction of active stars at larger distances above (and below) the Galaxy is significantly reduced; older stars have ceased being active. The decrease of active fractions as a function of vertical height above the Plane is seen for all $\mathrm{M}$ dwarf spectral types (W08).

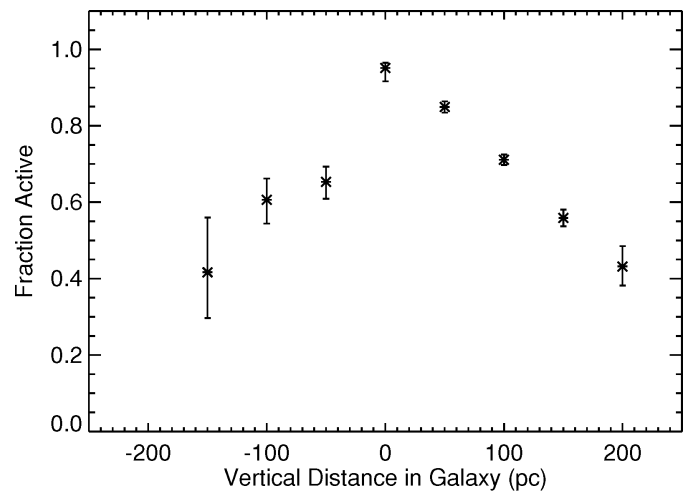

Figure 2. The fraction of active M7 stars as a function of vertical distance from the Galactic plane (reproduced from West et al. 2006). There is a significant decrease in the active fraction as a function of Galactic height, which can be used as a proxy for age. Younger stars near the Plane are almost all active, whereas older stars, further from the Plane have ceased being active. 
W08 also showed that the amount of activity, quantified by $L_{\mathrm{H} \alpha} / L_{\mathrm{bol}}$ (the ratio of the luminosity in the $\mathrm{H} \alpha$ emission line to the bolometric flux of the star) decreases over time. Figure 3 shows the median $\log \left(L_{\mathrm{H} \alpha} / L_{\mathrm{bol}}\right)$ as a function of vertical distance from the Plane. The narrow error bars represent the spread of the values and the wide error bars indicate the uncertainty of the median relation in each bin. The decrease as a function of height is a statistically significant over most of the spectral types. Some of the spectral types were omitted from Figure 3 because they lacked a sufficient number of active stars for a robust study.
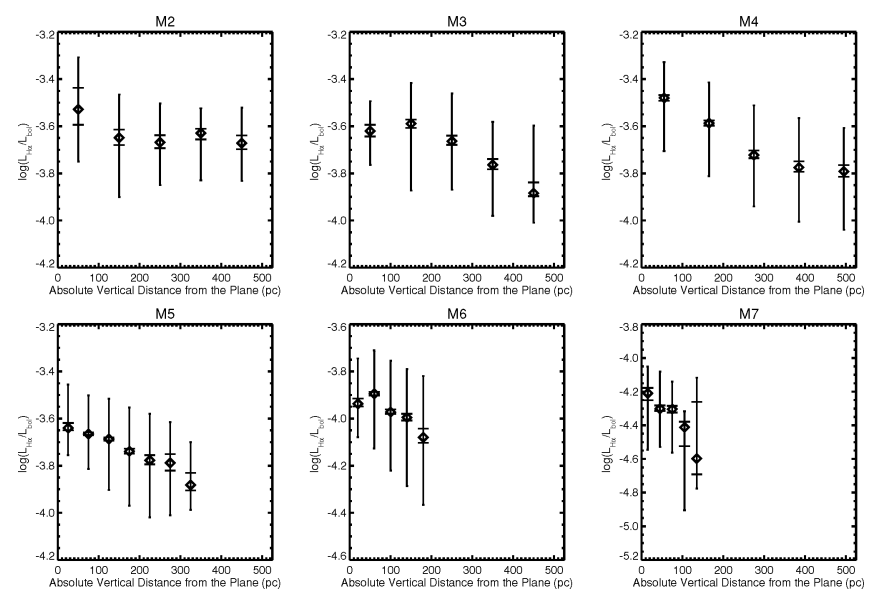

Figure 3. Median $\log \left(L_{\mathrm{H} \alpha} / L_{\mathrm{bol}}\right)$ as a function of vertical distance from the Plane (reproduced from W08). The narrow error bars represent the spread of the values and the wide error bars indicate the uncertainty of the median relation in each bin. The decrease as a function of height is a statistically significant over most of the spectral types.

\subsection{1-D dynamical model}

West et al. $(2006,2008)$ devolved a 1D dynamical model that traces the vertical dynamics of stars as a function of time. The model assumes a constant star formation rate and adds a new population of 50 stars every 200 Myr for a total simulation time of 10 Gyr. Each new population of stars begins with a randomly drawn velocity dispersion $8 \mathrm{~km}$ $\mathrm{s}^{-1}$ (Binney et al. 2000). Orbits are integrated using a "leap frog" integration technique (Press et al. 1992) and the vertical Galactic potential from Siebert et al. (2003).

Dynamical heating was simulated by altering the velocities of stars such that their velocity dispersions as a function of age match a $\sigma_{W} \propto t^{0.5}$ relation (Wielen 1977; Fuchs et al. 2001; Hänninen \& Flynn 2002; Nordström et al. 2004). Energy was added to stars that were within a certain distance from the Plane. This "region of influence" is a way to parameterize the cross section of interaction with molecular clouds during a Plane crossing (see West et al. 2006 for more information). The majority of the molecular gas is constrained to a small range of Galactic heights and the dynamical interaction depends on the proximity to the cloud, the density of the gas and the velocity of the star, all of which are absorbed in the "region of influence". The size of the "region of influence" was varied from \pm 0.5 to $\pm 5 \mathrm{pc}$ in intervals of $0.5 \mathrm{pc}$. Seventy simulations were run for each spectral type. Each simulation recorded the velocity, position, and age of each star.

\subsection{Age-activity}

W08 introduced a binary activity state to the dynamical models; stars started their lives as active and after a finite "lifetime", the activity turned off. The active lifetimes were 
varied from 0.0 to $9.0 \mathrm{Gyr}$ in $0.5 \mathrm{Gyr}$ intervals for each spectral type. The resulting model active fractions (as a function of vertical distance from the Plane) were compared to the empirical relations using a chi-squared minimization technique and a best-fit model for each spectral type was determined. The resulting $\mathrm{H} \alpha$ activity lifetimes are shown in Figure 4. The results from the Hawley et al. (2000) cluster study are overplotted for comparison (dotted). W08 found that there is a significant increase in activity lifetimes between spectral types M3 and M5, possibly indicating a physical change in the production of magnetic fields as the stellar interiors become fully convective.

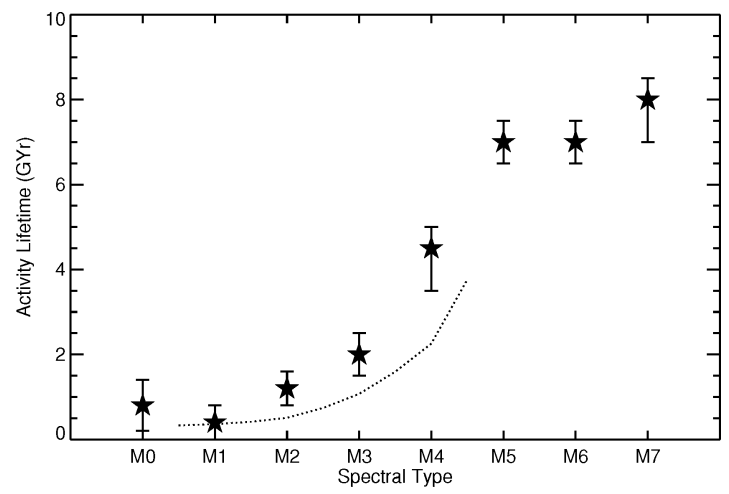

Figure 4. The $\mathrm{H} \alpha$ activity lifetimes of $\mathrm{M}$ dwarfs as determined by comparing the SDSS DR5 spectroscopic data to $1 \mathrm{D}$ dynamical simulations (stars; reproduced from W08). The Hawley et al. (2000) activity lifetime relation is overplotted for comparison. As predicted, the Hawley relation provides a lower limit to the ages. W08 found that there is a significant increase in activity lifetimes between spectral types M3 and M5, possibly indicating a physical change in the production of magnetic fields as full convection sets in.

Figure 3 suggests that activity is not simply a binary process, but rather that the amount of magnetic activity may decrease over time. To quantify this decrease, we compared the median $\log \left(L_{\mathrm{H} \alpha} / L_{\mathrm{bol}}\right)$ from Figure 3 to the median ages in the same vertical distance bins from the W08 1D dynamical models. The result, shown in Figure 5 , gives a statistical, quantitative relationship between the $\mathrm{H} \alpha$ activity of an $\mathrm{M}$ dwarf and it's age. Horizontal error bars represent the spread in age at a given bin. The ageactivity relations are consistent with a smooth Skumanich-like activity decrease until the star reaches its activity lifetime, after which it rapidly falls to its eternal inactive state.

We fit the activity-age relations in Figure 5 with a function of the form:

$$
\log \left(L_{\mathrm{H} \alpha} / L_{\mathrm{bol}}\right)=\frac{a}{l^{n}-t^{n}}-b
$$

where $a, b$ and $n$ are coefficients, $l$ is the active lifetime (Gyr), and $t$ is the age measured in Gyr. The exponent $n$ was forced to be the same for all spectral types. The lifetime $l$ coefficient was allowed to vary within the uncertainties of the derived active lifetimes from W08 (Figure 4). The resulting best-fit coefficients can be found in Table 1. We caution that these relations are purely statistical and may not be appropriate for use with individual stars. Additional discretion should be used with the functional fits, which may not be justifiably extrapolated beyond where data exist. 

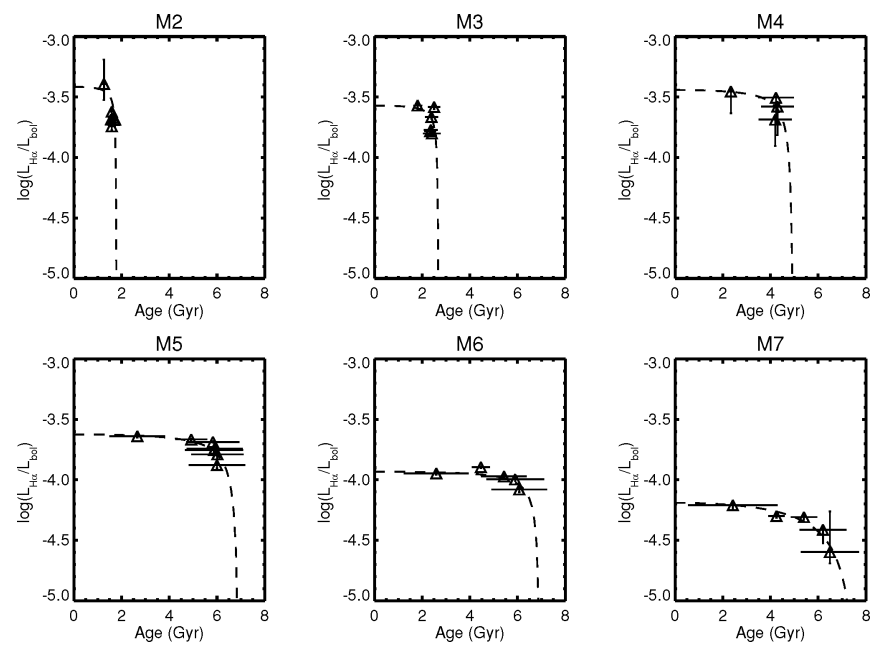

Figure 5. Median $\log \left(L_{\mathrm{H} \alpha} / L_{\mathrm{bol}}\right)$ as a function of age (as derived from the median age of the same bins in the W08 dynamical models). The error bars represent the spread in the distributions. The best-fit function is overplotted for each spectral type (dashed).

Table 1. Coefficients for best-fit activity-age relation

\begin{tabular}{ccccc}
\hline Spectral Type & $a$ & $b$ & $n$ & $l$ (Gyr) \\
\hline M2 & 0.106 & 3.38 & 2.0 & 1.8 \\
M3 & 0.213 & 3.54 & 2.0 & 2.7 \\
M4 & 1.41 & 3.39 & 2.0 & 5.0 \\
M5 & 2.85 & 3.57 & 2.0 & 7.0 \\
M6 & 1.78 & 3.90 & 2.0 & 7.0 \\
M7 & 11.8 & 4.01 & 2.0 & 8.0 \\
\hline
\end{tabular}

\section{Summary}

Large astronomical surveys have produced M dwarf samples of unprecedented size. We have shown that using the statistical foothold of the largest low-mass spectroscopic sample ever assembled, a strong tie between magnetic activity (as traced by $\mathrm{H} \alpha$ ) and age has been established and that $\mathrm{M}$ dwarfs have finite active lifetimes. These lifetimes are a strong function of spectral type, dramatically increasing as the $\mathrm{M}$ dwarf interiors become fully convective. We derived age-activity relations for M2-M7 dwarfs based on the average activity as a function of height above (or below) the Galactic plane. Future studies will extend these relations to other spectral types. In addition, these relations can be confirmed and calibrated using both white dwarf cooling ages in wide binary systems that have both a white dwarf and an M dwarf (see contributions by S. Catalan, M. Salaris, and J. Kalirai; Silvestri et al. 2006), and deep cluster observations of M dwarfs (when such observations become possible).

\section{References}

Barry, D. C. $1988, A p J, 334,436$

Binney, J., Dehnen, W., \& Bertelli, G. 2000, MNRAS, 318, 658

Bochanski, J. J., Munn, J. A., Hawley, S. L., West, A. A., Covey, K. R., \& Schneider, D. P. $2007 \mathrm{a}, A J, 134,2418$

Bochanski, J. J., West, A. A., Hawley, S. L., \& Covey, K. R. 2007b, AJ, 133, 531

Browning, M. K. 2008, ApJ, 676, 1262 
Chabrier, G. \& Baraffe, I. 1997, A\&A, 327, 1039

Covey, K. R., et al. 2007, AJ, 134, 2398

Delfosse, X., Forveille, T., Perrier, C., \& Mayor, M. 1998, A\&SA, 331, 581

Dobler, W., Stix, M., \& Brandenburg, A. 2006, ApJ, 638, 336

Eggen, O. J. 1990, PASP, 102, 166

Fleming, T. A., Schmitt, J. H. M. M., \& Giampapa, M. S. 1995, ApJ, 450, 401

Fuchs, B., Dettbarn, C., Jahreiß, H., \& Wielen, R. 2001, in ASP Conf. Ser. 228: Dynamics of Star Clusters and the Milky Way, ed. S. Deiters, B. Fuchs, A. Just, R. Spurzem, \& R. Wielen, 235

Giampapa, M. S. \& Liebert, J. 1986, ApJ, 305, 784

Gizis, J. E., Monet, D. G., Reid, I. N., Kirkpatrick, J. D., Liebert, J., \& Williams, R. J. 2000, AJ, 120,1085

Gizis, J. E., Reid, I. N., \& Hawley, S. L. 2002, AJ, 123, 3356

Gray, D. F. 1992, Science, 257, 1978

Hänninen, J. \& Flynn, C. 2002, MNRAS, 337, 731

Hawley, S. L., Covey, K. R., et al. 2002, AJ, 123, 3409

Hawley, S. L., Gizis, J. E., \& Reid, I. N. 1996, AJ, 112, 2799

Hawley, S. L., Reid, I. N., \& Tourtellot, J. G. 2000, in Very Low-mass Stars and Brown Dwarfs, Edited by R. Rebolo and M. R. Zapatero-Osorio. Published by the Cambridge University Press, UK, 2000., p.109, ed. R. Rebolo \& M. R. Zapatero-Osorio, 109-+

Hawley, S. L., Tourtellot, J. G., \& Reid, I. N. 1999, AJ, 117, 1341

Kiraga, M. \& Stepien, K. 2007, Acta Astronomica, 57, 149

Mamajek, E. E. \& Hillenbrand, L. A. 2008, ApJ, 687, 1264

Mohanty, S. \& Basri, G. 2003, ApJ, 583, 451

Munn, J. A., Monet, D. G., Levine, S. E., Canzian, B., Pier, J. R., Harris, H. C., Lupton, R. H., Ivezić, Z., Hindsley, R. B., Hennessy, G. S., Schneider, D. P., \& Brinkmann, J. 2004, AJ, 127,3034

Nordström, B., Mayor, M., Andersen, J., Holmberg, J., Pont, F., Jørgensen, B. R., Olsen, E. H., Udry, S., \& Mowlavi, N. 2004, A\&AA, 418, 989

Ossendrijver, M. 2003, A\&A Rev., 11, 287

Parker, E. N. 1993, ApJ, 408, 707

Pizzolato, N., Maggio, A., Micela, G., Sciortino, S., \& Ventura, P. 2003, A\&A, 397, 147

Press, W. H., Teukolsky, S. A., Vetterling, W. T., \& Flannery, B. P. 1992, Numerical recipes in C. The art of scientific computing (Cambridge: University Press, —c1992, 2nd ed.)

Reid, N. \& Hawley, S. L., eds. 2005, New light on dark stars : red dwarfs, low mass stars, brown dwarfs, ed. N. Reid \& S. L. Hawley

Reiners, A. \& Basri, G. 2007, ApJ, 656, 1121

Siebert, A., Bienaymé, O., \& Soubiran, C. 2003, A\&A, 399, 531

Silvestri, N. M., et al. 2006, AJ, 131, 1674

Skumanich, A. 1972, ApJ, 171, 565

Soderblom, D. R., Duncan, D. K., \& Johnson, D. R. H. 1991, ApJ, 375, 722

Stauffer, J. R., Liebert, J., Giampapa, M., Macintosh, B., Reid, N., \& Hamilton, D. 1994, AJ, 108,160

Thompson, M. J., Christensen-Dalsgaard, J., Miesch, M. S., \& Toomre, J. 2003, ARAA, 41, 599

West, A. A. \& Basri, G. 2009, ApJ, submitted

West, A. A., Bochanski, J. J., Hawley, S. L., Cruz, K. L., Covey, K. R., Silvestri, N. M., Reid, I. N., \& Liebert, J. 2006, AJ, 132, 2507

West, A. A., Hawley, S. L., Bochanski, J. J., Covey, K. R., Reid, I. N., Dhital, S., Hilton, E. J., \& Masuda, M. 2008, AJ, 135, 785

West, A. A., et al. 2004, AJ, 128, 426

Wielen, R. 1977, A\&A, 60, 263

Wilson, O. \& Woolley, R. 1970, MNRAS, 148, 463

York, D. G., et al. 2000, AJ, 120, 1579 


\section{Discussion}

R. Schiavon: How do you measure $\mathrm{H} \alpha$ ? (A boring question.)

A. West: That's not a boring question. We have to be careful about how we measure $\mathrm{H} \alpha$ and the pseudo-continuum because of the strong molecular features. We also require that an active star have $1 \AA$ equivalent width, which alleviates many problems. I would like to say we went through all 40,000 stars by eye, but we didn't. We have gone through a subset by eye and find we get $\mathrm{H} \alpha$ correct $96 \%$ of the time.

I. KING: It will be interesting to see your M-dwarf scale heights as a function of age. The empirical results will be so valuable because the theory is in such bad shape. Twenty years ago, when I was writing the dynamics chapter in The Milky Way As A Galaxy, I concluded that the theory of dynamical heating of the disk was in a very confused state, and I am not aware that it has been clarified in the two decades since.

A. West: Yes, we have compared our results to the empirical data of Nordström et al. (2004), West et al. (2006, 2008) and find excellent agreement. We have not however compared to theoretical predictions from star formation and dynamical theory.

B. WEAVER: With all these stars that you've measured, have you been able to determine a mass function for dwarf $\mathrm{M}$ stars.

A. West: Yes, our group at the University of Washington has derived two different luminosity and mass functions as part of the $\mathrm{PhD}$ theses of Kevin Covey (Covey et al. 2008, AJ, 136, 1778) and John Bochanski (PhD thesis, 2008; Bochanski et al. 2008, Proceedings of Cool Stars 15).

F. WALter: What is the relation between $L_{\mathrm{H} \alpha} / L_{\mathrm{bol}}$ and age for the active stars, to the left of where the activity plummets. Is it a Skumanich-like power law? Is there any dependence of the slope on the spectral type?

A. West: There appears to be a Skumanich-like decrease before the rapid decrease. This is difficult to see in the early types because they are probing ages near the active lifetimes but at M5 and M6, a plateau is quite clear. 
A. A. West et al.

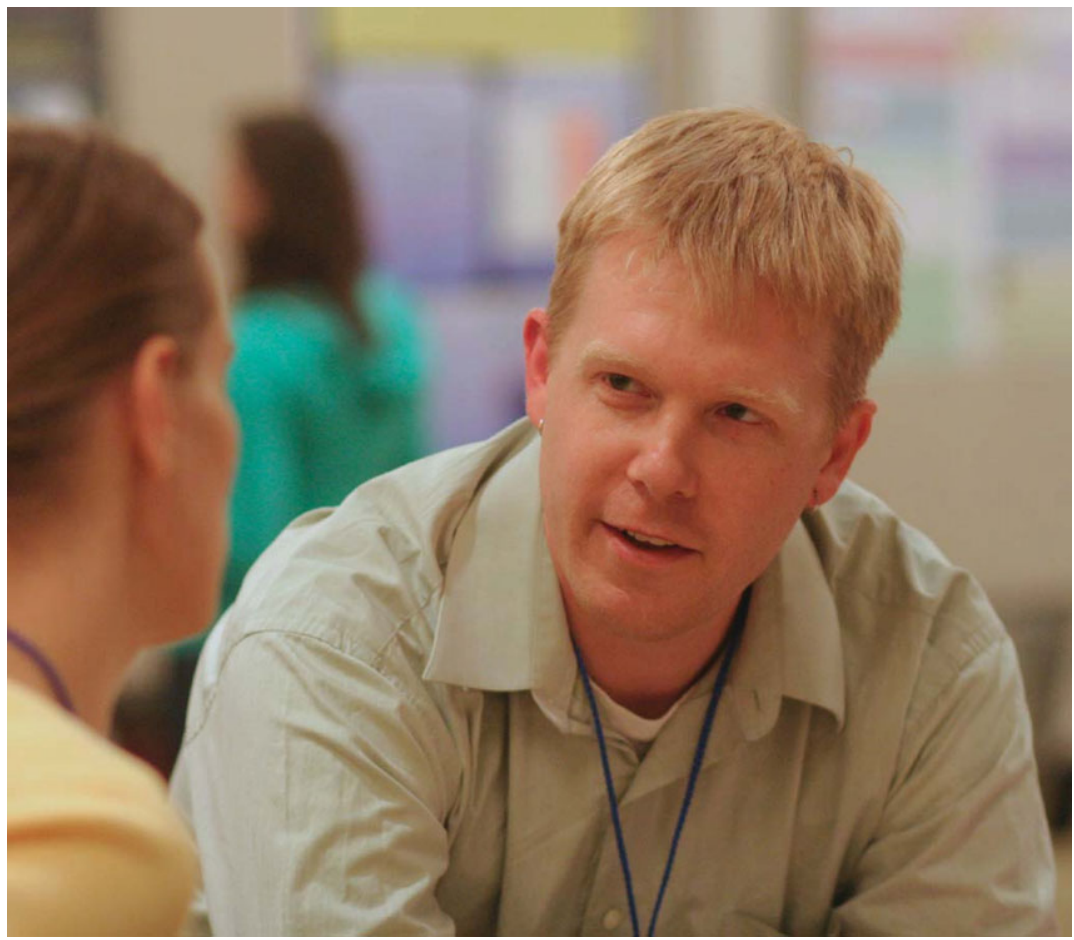

Andrew West

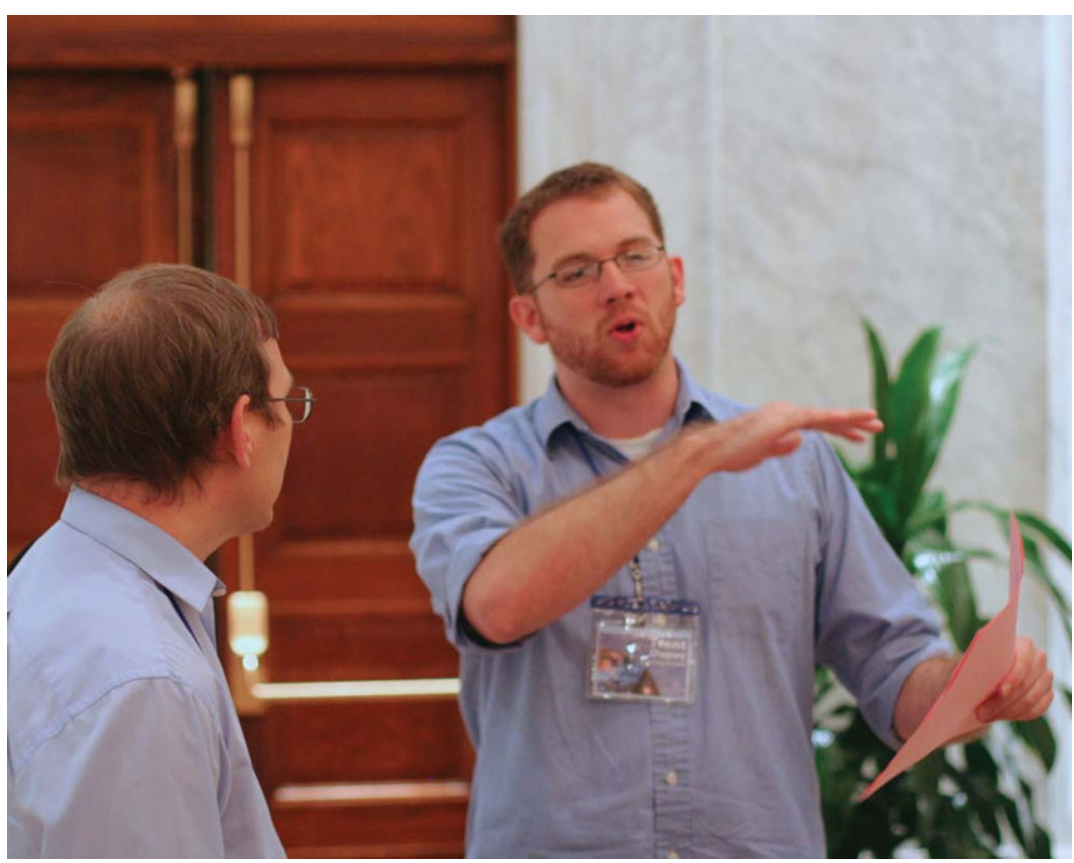

Trent Dupuy 